\title{
Gender Disparity in COVID-19 Case Severity
}

\author{
Shaun Lau ${ }^{1}$ and Elana Zizmor ${ }^{1}$ \\ ${ }^{1}$ Henry M. Gunn High School, Palo Alto, CA, USA

\section{ABSTRACT}

SARS-CoV-2 has made the world come to a halt, causing unprecedented global protocols and rules being implemented. Many different characteristics of this virus emerged early on. A notable characteristic that was noticed was the disparity in COVID-19 severity between the two biological genders, with males having a significantly higher fatality rate in comparison to females. As studies continue to understand this disparity, this literature review intends to summarize a few of these findings. Research on these studies were found on public research engines like ScienceDirect, Biology Medcentral, and PubMed. Papers were all peer reviewed. Another tool that was used was from the Global Health 50/50 research initiative which provided sex dis-aggregated data on COVID-19 in all countries that had accessible data. Studies have indicated that genetic factors, lifestyle factors, and "other" factors are all reasons for the disparity. T-Cell and ACE2 protein amount difference in males and females have been suggested as possible reasons for the discrepancy. Studies have also suggested that increased COVID-19 viral load also increases infection severity. This is relevant as male attitudes towards COVID-19 public health guidelines are more relaxed compared to females. Other possible factors that could play into this disparity is smoking, with active smokers having increased COVID-19 infection severity. This relates to the fact that the percent of male smokers is much higher than females. These lifestyle factors are further supported by the fact that males have higher fatality rates in respiratory tract diseases including SARS and influenza.

\section{Introduction}

The ongoing pandemic of SARS-CoV-2 has been an enigma for scientists, with many questions still without answers. The spread of the disease has no doubt put a burden on the healthcare system around the world, as many countries see infected individuals rise up at alarming rates. As of January 6th, 2021, the world has an estimated 87,015,526 cases and 1,878,581 deaths from COVID-19.

One question that has risen is the gender disparity of COVID-19 fatality rates. Several studies and data on this issue emerged early in the pandemic and continued to become more prominent. The research has strongly indicated that males have a significantly higher fatality rate in comparison to their biological counterparts. According to a June 2020 study in India, 71.4\% of the deaths from COVID-19 in the Southern Indian State of Tamil Nadu were males (Asirvatham et al, 2020). This data has been further supported by The Global Health 50/50 research initiative which has sex dis-aggregated data on COVID-19. According to its research, as of January 11th, 2021 the proportion of male to female deaths in confirmed cases in many of the world's hotspots seems to indicate that COVID-19 is more likely to prove fatal in males in comparison to females. As of January 11th 2021, the top 5 countries with the most fatalities from COVID-19 where sex-disaggregated data is available have the following COVID-19 male-to-female death ratio: U.S.A (1.27 male : 1 female), Brazil (1.06 male : 1 female), Mexico (1.71 male : 1 female), United Kingdom ( 1.49 male : 1 female), Italy (1.4 male: 1 female) (COVID-19 Sex-Disaggregated Data Tracker. 2020). The conclusion as of right now is that men face more severe consequences from COVID-19. Research that has been done on this topic have proposed several reasons for why men have a higher fatality rate in comparison to women when infected with the disease, including different body mechanisms, lifestyle choices, and immune system response to the disease. 


\section{Methodology}

Research was conducted on several public research engines including ScienceDirect, Biology MedCentral, and PubMed, with analysis on 19 papers. Furthermore, the initiative, Global Health 50/50, which provides sex-disaggregated data on COVID-19 with extensive tracking, was an imperative tool in obtaining information of the gender disparity, as its COVID-19 data tracker organizes national data of all countries, separating sex and age. Search terms for COVID19 related studies included COVID-19 gender differences, COVID-19 gender severity, COVID-19 gender impact. Information on Pneumonia and other respiratory disease fatalities were found on the CDC National Vital statistic reports, obtaining data from the year 2005, 2010, and 2015. Papers were all peer reviewed and relevant to the topic.

\section{Genetic factors affecting gender disparity in COVID-19 fatality rates}

\section{T-Cell Difference Between Male and Females:}

As studies have shown, it has been strongly suggested that females generally have stronger immune systems compared to males, and thus are innately better at defending against pathogens. These immune responses are factors in why males are more susceptible to COVID-19 in comparison to their biological counterparts. The first suggestion is the difference in T-cell activation between males and females. T-cells are a part of the immune system response that play an integral role in adaptive immunity, and thus in fighting off specific pathogens. According to a study which analyzed SARS-COV-2 patients at Yale-New Haven Hospital between March 18 - May 9, 2020, it showed a variety of immune responses that revealed a noticeable discrepancy between the two sexes. The study collected several samples including urine, saliva, swabs, and blood and conducted several experiments on them. A notable difference was in the amount of $\mathrm{T}$ cells between male and female patients (Takahashi et al, 2020). Female patients had an increase in activated $\mathrm{T}$ Cells $(\mathrm{P}$ value $=0.03$ ) in comparison to Male patients, most notably in the amount of activated CD38 T cells, PD-1, TIM-3 T cells, and HLA-DR-positive T cells, which were significantly increased in comparison to Male patients. Furthermore, results showed higher levels of certain cytokines and chemokines, including IL- 8 and IL-18, in male patients, another indication of an increase in severity in COVID-19 in male patients (Takahashi et al, 2020). Both Cytokine IL-8 and IL-18 are responsible for recruiting white blood cells and other immune cells at infection locations, therefore, an increase in these cytokines are a reflection of higher white blood cell count (Takahashi et al, 2020). It is noted however, that when adjusted for BMI and age, the analysis showed comparable levels of Cytokines IL-8 (p value $=0.44)$ and IL-18 $(\mathrm{P}$ value $=0.98)$ among male and female patients. The cytokine CCL5 however, was higher among male patients $(\mathrm{P}$ value $=0.02)$. Cytokine CCL5 is a type of chemokine which has pro-inflammatory effects (Culley et al, 2006), and therefore an increase in this Cytokine will cause a strong inflammatory response. Researchers also found that poorer activation of $\mathrm{T}$ cells increased with age for male patients but was not seen in female patients.

\section{Angiotensin Converting Enzyme 2 (ACE2) receptors in Male and Female:}

Another immune system response that plays a role in the discrepancy between male and female COVID patients is the ACE2 receptor (Yang et al, 2020). ACE2 are transmembrane enzymes located on both the cell membranes of a variety of organs, including, but not limited to: the lungs, stomach, small intestine, and brain (Hamming et al, 2020). The main function of ACE2 is to aid in blood regulation and facilitates amino acid transport. It also acts as a protective pathway for heart complications including hypertension, and lung disease (Gheblawi et al, 2020).

However, ACE2 is beneficial in that it has protective properties, especially in counteracting COVID-19. ACE2 plays a role in deactivating the renin-angiotensin system. This involves ACE2 converting Ang II, a peptide which increases blood pressure, blood volume, and also inflammatory responses, including cytokine pathways, into Ang (1-7), which is a peptide with anti-inflammatory effects, thus having protective properties. (Viveiros et al, 2020) (Benigni et al, 2020). 
ACE2 are also the primary receptors for SARS-COV and now SARS-COV-2, as the RNA virus's spike S1 protein binds to the ACE2 receptors (Mclachlan, 2020), which results in the viral entry into the cell, thus causing the infection. Studies have suggested that the mechanism of this binding involves the receptor binding domain of the spike S1 protein attaching to the tip of the ACE2 receptor. Membrane fusion then occurs between the virus and host cell, and viral RNA is released into the cytoplasm (Wentao et al, 2020). These studies have also indicated the ACE2 receptors as the reason for why infections are located in specific parts of the body. First, it explains that the ACE2 is mainly expressed in certain epithelial cells in the body that are consistent with where the virus mainly enters. One example being both type I and II alveolar epithelial cells (Wentao et al, 2020).

Recent studies have shown how differences in ACE2 expression in males and females could play a role in the severity of COVID-1. The ACE2 enzyme is an X-linked gene, which females have double of. Although one X chromosome tends to mostly be inactivated to prevent duplication, the ACE2 is an escape gene, and thus females have double the ACE2 (Viveiros et al, 2020). Furthermore, estrogen, the sex hormone found mainly in females, have been noted to activate and inhibit certain parts of the RES system, which the ACE2 acts on, and also helps upregulate ACE2 expression. Females therefore have an ACE2 upregulation mechanism when infected because of hormonal and genetic factors (Gagliardi et al, 2020).

As a result, studies have shown that this could be a possible factor in why males are more susceptible to COVID-19, giving females more protective properties against fighting the disease (Viveiros et al, 2020).

\section{Lifestyle factors affecting gender disparity in COVID-19 fatality rates}

\section{Smoking:}

Latest data has shown that smoking is a possible factor in the progression of COVID-19. Smoking can weaken the immune system and have effects all across the body (Centers for Disease Control and Prevention, 2020). Meta-analysis conducted by several researchers showed that there was weak data between smokers and risk of COVID-19 infection. Studies have indicated that this reason could be because of the upregulation of ACE2 expression found in smokers, although this has been challenged (Cai et al., 2020). As mentioned above, ACE2 are the host receptors for the SARSCoV-2 virus which leads to infection (Lippi \& Henry, 2020). However, unlike females, the increase in ACE2 expression for smokers makes these individuals more susceptible to the disease. This is due to the fact that females see an upregulation of ACE2 in response to infection, which has anti-inflammatory effects. In smokers however, the increased ACE2 expression makes it easier for the SARS-CoV-2 virus to bind to cells. This therefore suggests that there is a possible correlation between smoking and risk of SARS-CoV-2 infection (Lippi \& Henry, 2020). Several researchers have found that smokers had an increase in severity of COVID-19 in comparison to non-smokers. Through their meta-analysis, which included the characteristics of 11322 COVID-19 patients, certain correlations were found. Studies classified whether cases were severe or not by categorizing them. Cases that were severe meant: Critical, required ICU care, or fatal. Mild to moderate cases meant: common type, non-severe, did not require ICU care. According to the study, the prevalence of smokers in patients that were classified as mild to moderate COVID-19 was $2.9 \%$, whereas in severe cases it was 5.8\%. It also found that COVID-19 disease was severe in 10.7\% of nonsmokers, while it was severe in $21.2 \%$ of cases in active smokers $(\mathrm{P}=0.008)$. The analysis also found that the prevalence of patients that had a history of smoking was 5.2\% in non-severe cases and $12.5 \%$ in severe cases $(\mathrm{P}<0.001)$. An increase in COVID-19 severity can be seen in the data of patients who are active smokers and patients only with a history of smoking. Severe COVID-19 cases were approximately 2 times higher in patients who were active smokers compared to non-smokers and 2 times higher in patients with a history of smoking in comparison to patients without a history of smoking (Gülsen et al, 2020). According to the CDC, 15.3\% of Males in the U.S were current smokers in 2019, while $12.7 \%$ of females in the U.S were current smokers in 2019 (Centers for Disease Control and Prevention, 2019). The difference is greater worldwide, with data gathered by WHO in 2010 which have indicated that $40 \%$ of males were active smokers, compared to $9 \%$ of females. Thus, smoking could be a possible factor in why the prevalence of severe COVID-19 cases are higher in males in comparison to females. (World Health Organization, 2019) 


\section{Attitudes:}

Lifestyle choices and attitudes have also been suggested as a reason for why males are more susceptible to the virus in comparison to females. As studies have indicated that there is a correlation between the dosage of COVID-19 and the severity, there is a possible link between the attitudes of males towards COVID-19 and the discrepancy between the two biological genders in terms of severity of COVID-19. Studies conducted early in the pandemic have shown that males were less serious about COVID-19 in comparison to females. To obtain its data for the study, two surveys were sent out to 8 countries across the globe (U.S, UK, Australia, Austria, France, Germany, Italy, and New Zealand), one sent out on the 16 - 30 of March 2020, and another sent out on 15-20 April 2020. A total of 21,649 people responded for both surveys (Galasso et al, 2020). Respondents were asked how serious they were towards the health consequences of COVID-19, whether they agreed with public health policies, which include: closing school, limiting non-essential travel, closing non-essential businesses, imposing quarantine, and shelter-in-place. Respondents were also asked about their level of compliance to COVID-19 and its social distancing rules, which include wearing face masks, washing hands, social-distancing, and avoiding crowded places. According to its data, by mid-April, $40 \%$ of females considered COVID-19 a serious health problem, while 33\% of male correspondents considered it a serious health problem. Furthermore, by mid-April, survey data showed that $78 \%$ of females followed public-health recommendations, in comparison to $72 \%$ of males (Galasso et al, 2020). Males therefore took less precautions towards COVID-19 as opposed to females. This data is useful as we understand that there is a correlation in dosage of SARSCoV-2 and its severity.

\section{Other factors affecting gender disparity in COVID-19 fatality rates}

\section{Dosage of COVID-19:}

Researchers have also found that viral load of COVID-19 also has a positive correlation with the severity of the infection, thus also being a possible factor that affects the gender disparity in mortality rates. A recent study analyzed a total of 235 participants who were either hospitalized with COVID-19, considered symptomatic patients, or had recovered from the disease. The research was conducted through a thorough analysis of COVID-19 respiratory tract, plasma, blood, nasopharyngeal swabs and urine viral loads. Some limitations however were that a limited number of sputum tests due to many patients not able to generate a sample. Furthermore, the sample size of the number of participants was quite small and a larger study size would be needed in the future. The data observed that in each individual patient, showed a higher prevalence of the COVID-19 plasma viremia in hospital patients with severe symptoms in comparison to the other groups. Furthermore, the level of viremia prevalence also corresponded to the disease severity and degree of inflammation (Fajnzylber et al, 2020). Disease severity and degree of inflammation was also associated with low lymphocyte count, higher counts of Interleukin-6, increased levels of inflammatory cytokines IL8 , and increased levels of interferons. Research also discovered that patients with detectable plasma, nasopharyngeal, or sputum viral loads had a noticeable higher count of Interleukin and lower counts of lymphocyte compared to patients with undetected plasma, nasopharyngeal, or sputum viral loads (Fajnzylber et al, 2020). The data compared the plasma viremia of patients at the time of initial sampling, and the eleven participants who died, all had significantly higher levels of plasma viremia. This is imperative as it could link towards the gender disparity in the severity of COVID-19. As discussed later, male attitudes towards COVID-19 are less serious than females, and that a smaller percentage of males follow public health guidelines in comparison to females. This enhances the possibility of increased viral dose when exposed to COVID-19 for males, which possibly then correlates to an increase in severity of symptoms when infected with COVID-19. Therefore, could be a possibility in why males are more susceptible to COVID-19 in comparison to females. 


\section{In Line with Other Respiratory Tract Diseases:}

The trend of Men being more susceptible to respiratory tract diseases has been apparent in other coronavirus outbreaks, such as SARS (Severe Acute Respiratory Syndrome) and MERS (Middle East Respiratory Syndrome). Data extracted from the SARS pandemic between early March to late September 2003 showed that Males had a $21.9 \%$ fatality rate compared to females who had a $13.2 \%$ fatality rate $(\mathrm{P}<0.0001)$ (Karlberg et al, 2020), therefore strongly suggesting that Males faced more severe effects from SARS.

This is somewhat similar to what is seen in MERS, as data between 2012-2019 in Saudi Arabia shows that males made up $70.07 \%$ of fatalities, while females made up $29.93 \%$ of fatalities. (Khan et al, 2020). However, it must be noted that males made up $69.1 \%$ of infected patients, while females made up $30.9 \%$, indicating mainly a strong disparity between the number of cases rather than mortality rates. Note that this data was similar to a 2015 data analysis of MERS cases in South Korea, which saw a ratio of 1.70:1.

Another infection that follows this trend is seasonal influenza. According to the National Vital Statistic reports of 2005, 2010, and 2015, age-adjusted deaths caused by influenza/pneumonia all have displayed a higher death ratio among males in comparison to females. The ratio between male-to-female for 2005, 2010, and 2015 were (1.3: 1), (1.4:1), (1.3:1), respectively (Kung, 2005; Murphy, 2010; Murphy, 2015). The parallel between the respiratory diseases have strongly signified that there is a noticeable disparity in respiratory tract disease deaths between males and females and suggests that men are more susceptible to general respiratory diseases in comparison to females, not just with SARS-CoV-2.

\section{Conclusion}

COVID-19 continues to affect millions of lives across the world. The spread of this virus continues to be rapid, due to its complexity and its unpredictable severity of infection from person to person. The mechanisms and components of the COVID-19 virus will continue to be studied for many years to come, which will help answer the questions of SARS-CoV-2. As the mass rollout of vaccines continues in many countries, other therapeutic agents could be developed to further fight against this virus. However, these therapeutic agents would have to adjust to the unpredictable severity levels of the virus for different patients. Many different factors seem to play into the severity level, including gender. COVID-19 has had a higher fatality rate on Male in comparison to their biological counterparts. Through meta-analysis of studies and research that has been done on this topic, a series of explanations could help us in understanding the disparity that is going on. It can be argued that all these explanations could factor into why males face more serious symptoms when infected with COVID-19 in comparison to females.

Difference in Immune response, and lifestyle/attitudes towards COVID-19 are all likely factors in the discrepancy in case severity between males and females. The ACE2 (Angiotensin Converting Enzyme 2) receptor, which is the host receptor cell for the SARS-CoV and SARS-Cov-2 virus, was a factor in the discrepancy of both nonsmokers/smokers and male/female patients in terms of severity of COVID-19 infection. This is largely due to the protein's benefits and disadvantages. While an increase in ACE2 causes more susceptibility to COVID-19, an upregulation during infection is beneficial due to ACE2's blockage of the RAS pathway, and producing anti-inflammatory effects. Thus, research into ACE2 and steps we can take with the protein would be vital for combatting different viruses in the future.

The gender discrepancy in case severity for COVID-19 infections is important because it gives a better understanding on different treatment methods and establishing that there is a difference will further aid in combating future viruses. To add on, it gives us a better understanding of the immune system and its response to pathogens, which will aid in future development of therapeutics and other medicine. 


\section{Limitations}

Limitations in some of these studies are that the sample size of the findings were quite small for a few of the studies. Specifically, the Research at Yale-New Haven Hospital on viral dosage. The study sample was only 235, and so future studies with a larger study size would be needed. Another limitation was the study of the gender difference in attitudes towards COVID-19 (Galasso et al, 2020). The survey was conducted in March 2020 and April 2020, and as the disease has still persisted in many countries, attitudes towards the virus have possibly changed. One other limitation is that SARS-CoV-2 is still a fairly new disease and so many studies on this virus will need to be conducted in the future in order to have conclusive evidence to support or disregard the notions that have been suggested into why there is a discrepancy in COVID-19 infection severity between males and females.

\section{Acknowledgments}

I would like to thank and give my appreciation to Ms. Elana Zizmor for helping me with this project.

\section{References:}

1. Asirvatham, E., Sarman, C., Saravanamurthy, S., Mahalingam, P., Maduraipandian, S., \& Lakshmanan, J. (2020, October 03). Who is dying from COVID-19 and when? An Analysis of fatalities in Tamil Nadu, India. Retrieved January 23, 2021, from https://www.sciencedirect.com/science/article/pii/S2213398420302189

2. COVID-19 Sex-Disaggregated Data Tracker. (2020). Retrieved January 23, 2021, from https://globalhealth5050.org/the-sex-gender-and-covid-19-project/dataset/

3. Takahashi, T., Ellingson, M., Wong, P., Israelow, B., Lucas, C., Klein, J., . . Iwasaki, A. (2020, August 26). Sex differences in immune responses that underlie COVID-19 disease outcomes. Retrieved January 23, 2021, from https://www.nature.com/articles/s41586-020-2700-3

4. Culley, F., Pennycook, A., Tregoning, J., Dodd, J., Walzl, G., Wells, T., . . Openshaw, P. (2006, August 15). Role of CCL5 (RANTES) in Viral Lung Disease. Retrieved January 28, 2021, from https://jvi.asm.org/content/80/16/8151

5. Yang, J., Petitjean, S., Koehler, M., Zhang, Q., Dumitru, A., Chen, W., . . Alsteens, D. (2020, September 11). Molecular interaction and inhibition of SARS-CoV-2 binding to the ACE2 receptor. Retrieved January 23, 2021, from https://www.nature.com/articles/s41467-020-18319-6

6. Hamming, I., Timens, W., Bulthuis, M., Lely, A., Navis, G., \& Van Goor, H. (2004, June). Tissue distribution of ACE2 protein, the functional receptor for SARS coronavirus. A first step in understanding SARS pathogenesis. Retrieved January 25, 2021, from https://www.ncbi.nlm.nih.gov/pmc/articles/PMC7167720/

7. Gheblawi, M., Wang, K., Viveiros, A., Nguyen, Q., Zhong, J., Turner, A., . . Oudit, G. (2020, May 8). Angiotensin-Converting Enzyme 2: SARS-CoV-2 Receptor and Regulator of the Renin-Angiotensin System: Celebrating the 20th Anniversary of the Discovery of ACE2. Retrieved January 23, 2021, from https://www.ncbi.nlm.nih.gov/pmc/articles/PMC7188049/ 
8. Viveiros, A., Rasmuson, J., Vu, J., Mulvagh, S. L., Yip, C. Y., Norris, C. M., \& Oudit, G. Y. (2020, December 4t). Sex differences in COVID-19: Candidate pathways, genetics of ACE2, and sex hormones. Retrieved January 7th, 2021, from https://journals.physiology.org/doi/pdf/10.1152/ajpheart.00755.2020

9. Benigni, A., Cassis, P., \& Remuzzi, G. (2010, July). Angiotensin II revisited: New roles in inflammation, immunology and aging. Retrieved January 23, 2021, from https://www.ncbi.nlm.nih.gov/pmc/articles/PMC3377325/

10. McLachlan, C. (2020, July 15). The angiotensin-converting enzyme 2 (ACE2) receptor in the prevention and treatment of COVID-19 are distinctly different paradigms. Retrieved January 23, 2021, from https://www.ncbi.nlm.nih.gov/pmc/articles/PMC7360378/

11. Ni, W., Yang, X., Yang, D., Bao, J., Li, R., Xiao, Y., . . Gao, Z. (2020, July 13). Role of angiotensin-converting enzyme 2 (ACE2) in COVID-19. Retrieved January 5, 2021, from https://ccforum.biomedcentral.com/articles/10.1186/s13054-020-03120-0\#citeas

12. Gagliardi, M., Tieri, P., Ortona, E., \& Ruggieri, A. (2020, May 26). ACE2 expression and sex disparity in COVID-19. Retrieved January 23, 2021, from https://www.nature.com/articles/s41420-020-0276-1

13. Karlberg, J., Chong, D., \& Lai, W. (2004, February 01). Do Men Have a Higher Case Fatality Rate of Severe Acute Respiratory Syndrome than Women Do? Retrieved January 23, 2021, from https://academic.oup.com/aje/article/159/3/229/79939

14. Khan, S., El Morabet, R., Khan, R., Bindajam, A., Alqadhi, S., Alsubih, M., \& Khan, N. (2020, December 10). Where we missed? Middle East Respiratory Syndrome (MERS-CoV) epidemiology in Saudi Arabia; 2012-2019. Retrieved January 23, 2021, from https://www.ncbi.nlm.nih.gov/pmc/articles/PMC7398055/

15. Fajnzylber, J., Regan, J., Coxen, K., Corry, H., Wong, C., Rosenthal, A., . . Li, J. (2020, October 30). SARS-CoV-2 viral load is associated with increased disease severity and mortality. Retrieved January 23 , 2021, from https://www.nature.com/articles/s41467-020-19057-5

16. Cai, G., Bossé, Y., Xiao, F., Kheradmand, F., \& Amos, C. (2020, June 15). Tobacco Smoking Increases the Lung Gene Expression of ACE2, the Receptor of SARS-CoV-2. Retrieved January 23, 2021, from https://www.atsjournals.org/doi/pdf/10.1164/rccm.202003-0693LE

17. Centers for Disease Control and Prevention. (2020, December 10). Health Effects of Cigarette Smoking. Retrieved April 26, 2021, from https://www.cdc.gov/tobacco/data_statistics/fact_sheets/health_effects/effects_cig_smoking/index.htm\#other-health-risks.

18. Lippi, G., \& Henry, B. (2020, May). Active smoking is not associated with severity of coronavirus disease 2019 (COVID-19). Retrieved January 23, 2021, from https://www.ncbi.nlm.nih.gov/pmc/articles/PMC7118593/

19. Gülsen, A., Yigitbas, B., Uslu, B., Drömann, D., \& Kilinc, O. (2020, September 8). The Effect of Smoking on COVID-19 Symptom Severity: Systematic Review and Meta-Analysis. Retrieved January 23, 2021, from https://www.ncbi.nlm.nih.gov/pmc/articles/PMC7499286/ 
20. Pampel, F. (2006, December). Global Patterns and Determinants of Sex Differences in Smoking. Retrieved January 23, 2021, from https://www.ncbi.nlm.nih.gov/pmc/articles/PMC3160810/

21. Galasso, V., Pons, V., \& Profeta, P. (2020, November 7). Gender differences in COVID-19 perception and compliance. Retrieved January 23, 2021, from https://voxeu.org/article/gender-differences-covid-19-perception-and-compliance

22. Centers for Disease Control and Prevention. (2019) Current Cigarette Smoking Among Adults in the United States. Retrieved January 23 2021, from https://www.cdc.gov/tobacco/data_statistics/fact_sheets/adult_data/cig_smoking/index.htm

23. World Health Organization (2010) World Health Organization, from https://www.who.int/gender/documents/10facts gender tobacco en.pdf

24. (2005)Kung, H., Hoyert, D. L., Xu, J., \& Murphy, S. L. (2005). Deaths: Final Data for 2005 (Vol. 56, pp. 5-6, Rep. No. 10). CDC. https://www.cdc.gov/nchs/data/nvsr/nvsr56/nvsr56 10.pdf

25. (2010) Murphy, S. L., Xu, J., \& Kochanek, K. D. (2013). Deaths: Final Data for 2010 (Vol. 61, pp. 5-6, Rep. No. 4). CDC. https://www.cdc.gov/nchs/data/nvsr/nvsr61/nvsr61_04.pdf

26. (2015) Murphy, S. L., Xu, J., Kochanek, K. D., Curtin, S. C., \& Arias, E. (2017). Deaths: Final Data for 2015 (Vol. 66, pp. 5-6, Rep. No. 6). CDC. https://www.cdc.gov/nchs/data/nvsr/nvsr66/nvsr66_06.pdf 